JHR

35,6

Received 8 November 2019 Revised 11 February 2020 Accepted 25 March 2020

\section{Resiliency and mental health of school teachers in Okinawa}

\author{
Paul Ratanasiripong \\ College of Education, California State University, Long Beach, California, USA \\ Takashi China \\ Okinawa International University, Ginowan, Japan \\ Nop T Ratanasiripong \\ School of Nursing, California State University-Dominguez Hills, Carson, \\ California, USA, and \\ Shiho Toyama \\ California State University, Long Beach, California, USA
}

\begin{abstract}
Purpose - The purpose of this paper is to describe the mental health issues among teachers globally and to investigate the significant factors that specifically impact the mental health of school teachers in Okinawa, Japan.

Design/methodology/approach - This cross-sectional study examined depression, anxiety, stress, selfesteem and resiliency among 174 teachers from seven schools in Okinawa, Japan. The study questionnaire consisted of four parts, including demographic data, Depression, Anxiety, and Stress scale (DASS-42), Rosenberg Self-Esteem Scale (RSE), and Connor-Davidson Resiliency Scale (CD-RISC). Multiple regression analyses were performed to identify predictors of mental health variables.

Findings - Of the 174 teachers, 111 were females (64\%) and 60 were males (35\%). Average age of participants was $41.65(\mathrm{SD}=10.07)$. Average number of years being a teacher was $15.50(\mathrm{SD}=9.88)$. There was a significant gender difference in the level of self-esteem. Significant differences in anxiety were found among varying grade levels taught. Regression analyses indicated that resiliency and self-esteem significantly predicted depression, anxiety and stress among school teachers in Okinawa.

Originality/value - This is the first study among school teachers in Okinawa that examined the impact of resiliency and self-esteem on their mental health. To reduce psychological distress common within the teaching profession, social and environmental support should be provided within the school to better foster the successful promotion of teacher resiliency and self-esteem.
\end{abstract}

Keywords Resilience, Depression, Anxiety, Stress, Teachers, Japan

Paper type Research paper

\section{Introduction}

Teachers worldwide demonstrate susceptibility to mental health issues such as depression, anxiety and stress based on the multitude of primary stressors they encounter, including a lack of resources, a pressure to fulfill multiple roles, interpersonal troubles, demanding working conditions, low student achievement and excessive workload [1-5]. Further, numerous studies have demonstrated that these primary stressors threaten the psychological wellbeing of teachers internationally [6-10]. Poor psychological wellbeing may contribute to problems such as low teacher performance and teacher burnout, which in turn may lead to

(C) Paul Ratanasiripong, Takashi China, Nop T Ratanasiripong and Shiho Toyama. Published in Journal of Health Research. Published by Emerald Publishing Limited. This article is published under the Creative Commons Attribution (CC BY 4.0) licence. Anyone may reproduce, distribute, translate and create derivative works of this article (for both commercial and non-commercial purposes), subject to full attribution to the original publication and authors. The full terms of this licence may be seen at http:// creativecommons.org/licences/by/4.0/legalcode
Journal of Health Research Vol. 35 No. 6,2021 pp. $470-481$

Emerald Publishing Limited e-ISSN: 2586-940X p-ISSN: 0857-4421 DOI 10.1108/JHR-11-2019-0248 
costly social and economic consequences, such as poor academic outcomes for students and low retention rates of quality teachers [8,11-14]. An improved understanding of factors contributing to teacher wellbeing is therefore necessary for increasing the longevity and quality of teachers across the globe.

In the general population, resilience has consistently demonstrated a negative correlation with mental health issues [15-18]. However, limited research has examined teacher resilience and its impact on teachers' psychological wellbeing. This study examines mental health and resilience among Okinawan teachers. Understanding the relationship between resilience and mental health issues in this subgroup of teachers may offer valuable insights into the mental health of teachers in the international context.

\section{Resilience and self-esteem}

Resilience is defined as an individuals' ability to positively adapt to stressful situations [19]. Resilient individuals tend to successfully adapt to the exposures of stressors, thus decreasing maladaptive responses and negative consequences [20]. In turn, high levels of resilience correspond to positive psychological wellbeing [16,17,19,21-23]. A study in the United States found that resilient coping was negatively correlated with depression and neuroticism [17]. Further, resilient coping was identified as a moderator between depression and trauma exposure; among individuals who experienced trauma, depression levels were roughly twice as high for those with lower resilient coping skills, illustrating the positive effect of resilience on depression [17]. More importantly, resilience was found to be a predictor of depression, work stress and burnout among diverse sample populations, exhibiting a strong influence on mental health issues [24-26]. Adding to this notion, a study in Australia found that resilience was the only variable that decreased the probability of clinical depression [23].

The impact resilience has on multiple mental health issues may be explained by elevated levels of self-esteem among resilient individuals; resilience was positively correlated with selfesteem in Turkey [15]. Self-esteem, defined as the attitude an individual has regarding one's self-concept, determines the individual's beliefs in their abilities. Individuals with high selfesteem demonstrate their ability to utilize coping strategies to mitigate or eliminate experienced stressors, increasing resilience among individuals with high self-esteem [27]. Thus, resilience and self-esteem play a vital role in psychological wellbeing among individuals.

\section{Mental health of teachers globally}

Multiple studies have demonstrated the ubiquity of mental health issues experienced by teachers internationally. A study involving secondary school teachers in Malaysia indicated severe depression and stress among 3\% and severe anxiety among $13 \%$ of the sample population [28]. A study on Italian teachers reported $11 \%$ anxiety and $49 \%$ depression, distinguishing work stress as the major factor for depression and anxiety among teachers [6]. In addition to the stressors faced by teachers during school hours, teachers are required to generate lesson plans prior to class lectures and spend additional post-class hours grading paperwork, resulting in roughly 50 work hours per week and two-thirds of total work time per month spent on overtime [5,29]. This contributes to teachers' global vulnerability to mental health issues. A study of female elementary school teachers in Brazil concluded that teachers who worked the longest hours had the highest prevalence of depression [7].

Although depression, anxiety and stress are established issues among the global teaching population, specific factors impact teachers' mental health to varying degrees. Teachers in rural areas have higher odds of depression than teachers in urban schools [30]. In addition, elementary school teachers in the United States reported the highest level of major depressive disorder at $47 \%$ in comparison to high school and middle school teachers with 27 and $22 \%$,

Resiliency and mental health of teachers 
JHR 35,6

respectively [31]. Similarly, a study of Chinese teachers indicated that primary school, middle school and special education teachers reported worse levels of mental health than kindergarten teachers and college instructors [32].

\section{Mental health in Okinawa}

According to World Mental Health, Japan had lower levels of mental health issues than most other participating countries in North and Southeast Asia [33]. However, Japanese citizens still demonstrate concerning levels of mental health issues, reporting over 5.7 million cases of depressive disorders and roughly 3.7 million cases of anxiety disorders in 2015, with anxiety disorders declared the most common in a 12-month period [34]. An estimated 2.6 million Japanese suffered from role impairment due to mental disorders, suggesting the impact of mental health issues on life functions [33].

However, prefectural differences revealed varying discrepancies in the psychological wellbeing of Japanese populations. More specifically, the Okinawa prefecture has exhibited unique mental health trends uncommon among populations across the globe. Generally, low socioeconomic status is correlated with higher levels of depression [35,36]. However, Okinawa - ranking lowest in prefectural income - indicated a low prevalence of patients with major depressive disorder, ranking 35th out of the 47 prefectures [37]. Additionally, gender differences have been exhibited among the Okinawan population. Female Okinawans ranked 44th in suicide victims, illustrating a low prevalence of suicide and better mental health in comparison to their male counterparts which ranked 16th [37]. Males may be more reluctant to express emotional needs; accumulated experiences of mental health issues may, therefore, increase the vulnerability to suicide among the male Okinawan population.

The distinction between prevalence and influences of mental health issues within Okinawa may be explained by the complex history of Okinawa and its geographic location. Okinawa began as an independent country ruled by the Ryukyu kingdom [38]. However, in 1609, the Satsuma Kingdom invaded Okinawa, forcing Okinawa to join the rest of Japan under the Shogunate system; in 1879, the establishment of the prefecture system occurred, turning Okinawa into one of the 47 prefectures of Japan [38]. Despite its recognition as a prefecture of Japan, the end of the Pacific War in 1945 marked another period in history when Okinawa was no longer part of Japan; instead, Okinawa was placed under the U.S. administration, only to return to the Japanese administration in 1972 [38]. Throughout this course of history, Okinawa has been exposed to various populations and rulers that influence the overall culture established within this particular prefecture. Additionally, Okinawa is in closer proximity to China in comparison to mainland Japan, and thus inherits Confucian beliefs commonly practiced within Chinese cultures [39]. The variability highlighted throughout the historical and geographic background of Okinawa may in part explain the distinct mental health issues that the Okinawan population experience.

Limited literature is available on the mental health of Okinawan residents and even less literature has been published on the psychological wellbeing of Okinawan teachers. A comparison study between the general Japanese population and Japanese teachers did not demonstrate a significant difference in depression scores, implying that Japanese teachers may show similar trends in depression as the general population [40]. Teachers in Japan are considered equals and salary is based strictly on accumulated service years, thus eliminating the confusion and anxiety presented by the differences in pay rate between themselves and their colleagues [41]. However, teachers were more stressed and possessed more negative subjective moods than other white-collar workers. This may be a result of the additional responsibilities teachers take on without compensation. As students in Japan become heavily involved in extracurricular activities during middle and high school education, teachers are required to dedicate additional hours in their career to plan and lead such activities with no 
additional compensation [42,43]. The negative subjective mood frequently experienced by Japanese teachers may be detrimental to teacher's perception of the work environment and attitude, accounting for $88 \%$ of reported severe or very severe depressive symptoms among teachers $[40,41,44]$. Supporting this notion, $63 \%$ of elementary and junior high school teachers reported major psychiatric disorders contributed by factors such as job dissatisfaction and reduced leisure time [45]. Although working long hours was not considered a risk factor, reduced leisure time may be a potential consequence of longer work hours, thus paralleling results of global studies [5,9,46,47].

Teaching is considered a prestigious job in Japan, and the wider society holds high expectations of teachers [48]. Further, the structure of the Japanese educational system increases the stress experienced by Japanese teachers. Only the first nine years of school, consisting of six years in elementary school and three years in middle school, are considered compulsory [43]. Students who wish to continue their education are required to pass an entrance exam to be admitted into the high school of their choice [43]. The pressures to perform within a prestigious profession and maintain the standards and academic outcomes of students within their affiliated institution heighten teacher vulnerability to mental health issues [49].

In addition to these public standards, the dominant Eastern culture's emphasis on outside approval enumerates the stress distinctly experienced by Japanese teachers. Stress among Japanese teachers is often experienced through interpersonal relationships with colleagues $[48,50]$. In other words, relationships with colleagues, supervisors and other personnel within teachers' academic groups are more highly associated with stress among Japanese teachers in comparison to their relationship with their students [50,51]. In 2015, Japanese teachers worked 1,891 hours in the year, roughly 200 more hours than the average teacher across the globe [42]. Experienced teachers are required to provide mentorship to new teachers undergoing an induction period, without formal training or compensation for the additional responsibilities [42]. Both mentee and mentor are required to work together on classroom management, subject guidance, lesson planning, and analyzing classroom teaching, resulting in increased workload and interactions with their colleagues and potentially decreasing their psychological wellbeing $[5,42]$.

Gender differences were also prevalent among the Japanese teaching population. As observed globally, male teachers in Japan have better mental health and higher self-esteem than female teachers [52,53]. Female teachers experience supplementary stressors, such as household responsibilities which impact their mental health and can result in less time to prepare for teaching and fulfill other job demands. These problems contribute to the lower psychological wellbeing of female teachers as illustrated among female teachers worldwide [45,52].

A significant gap in the literature exists on the resilience and mental health of school teachers in Okinawa. Additionally, the Japanese educational system is divided based on prefecture; each prefecture has different municipalities and school boards [42]. Therefore, it is important to examine the mental health of teachers in Okinawa as a separate entity to mainland Japan and its prefectures. Determining the relationship between resilience and mental health issues among the Okinawa teacher population will help identify pertinent strategies for improving teachers' collective mental health. Based on previous research, the conceptual framework for this study is displayed in Figure 1. Specifically, among school teachers in Okinawa, resilience and self-esteem are hypothesized to be significant predictors for depression, anxiety and stress.

\section{Methods}

\section{Procedures and participants}

This cross-sectional study used a convenience sample. After Human Ethics Board approval (CSULB Ref\#19-108), several public schools in Okinawa prefecture were invited to
Resiliency and mental health of teachers 
JHR

474

Figure 1.

Conceptual framework for depression, anxiety, and stress

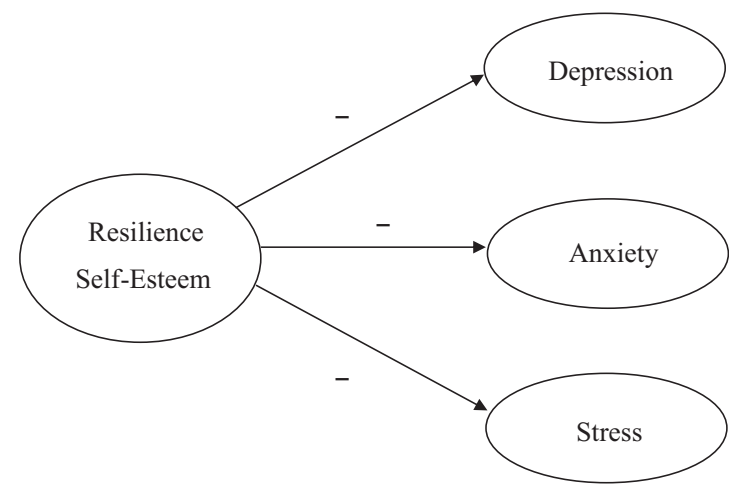

participate in the study. Seven schools responded to the invitation. The paper-based surveys were distributed to all the teachers at each school. To be eligible for the study, the participant had to be employed as a teacher at the school. Teachers who volunteered for the study and agreed to the informed consent completed the anonymous survey that included demographic data and three instruments.

\section{Measurements}

The Japanese version of the Depression, Anxiety, Stress Scale (DASS) was used to measure the levels of depression, anxiety and stress [54]. The Japanese DASS had 42 statements (14 per subscale) for participants to rate, from 0 (did not apply to me at all) to 3 (applied to me most of the time). Higher scores indicated more symptoms. For this study, Cronbach's alpha was 0.91 for depression, 0.83 for anxiety, and 0.91 for stress.

The Japanese version of the Connor-Davidson Resilience Scale (CD-RISC) was used to assess the level of resilience [55]. The Japanese CD-RISC had 25 statements for participants to rate, from 0 (not true at all) to 4 (true nearly all the time). A higher score indicated a higher level of resilience. Cronbach's alpha of the CD-RISC was 0.94 for this study.

The Japanese version of the Rosenberg Self-Esteem Scale (RSE) was used to measure the level of self-esteem [56]. The Japanese RSE had 10 statements for the participant to rate, from 1 (disagree) to 4 (agree). Higher scores indicated higher self-esteem. Cronbach's alpha for the RSE for this study was 0.86 .

\section{Statistical analyses}

Statistical analyses were performed by SPSS version 24. Between-group comparisons were done using independent sample $t$-tests (normality and homogeneity of variance assumptions were checked). Pearson's correlations were utilized to explore associations between variables of interests (depression, anxiety, stress, self-esteem, and resilience). Depending on the number of potential predictors, simple or multiple linear regression analysis was used to identify predictors for each mental health issue. The statistically significant level was set at $p<0.05$. To check the minimal number of subjects required for multiple regression analyses, the parameters of effect size $=0.15$, power $=0.9$, alpha $=0.05$, and significantly related predictors (self-esteem, resilience, and class level, for anxiety) $=3$ were entered in $\mathrm{G}^{*}$ Power 3.1 [57]. The 0.15 effect size $\left(f^{2}\right)$ was calculated from the squared multiple correlation $p^{2}$ in $\mathrm{G}^{*}$ Power. The minimum number of subjects needed was 99. 


\section{Results}

Out of 200 teachers from seven public schools, 174 teachers participated in this study $(87 \%$ response rate). There were 111 female teachers (64\%) and 60 male teachers (35\%). There were 108 primary school teachers $(62 \%)$ and 66 secondary school teachers $(38 \%)$. Average age of participants was $41.65(\mathrm{SD}=10.07$, Range $=22-62)$. The average number of years being a teacher was $15.50(\mathrm{SD}=9.88$, Range $=1-40)$. Average class size was $27(\mathrm{SD}=10.42$, Range $=2-40)$.
Resiliency and mental health of teachers

\section{Significant associations of the variables}

Among school teachers in Okinawa, self-esteem in male teachers $(M=30.97, \mathrm{SD}=4.90)$ was significantly higher than female teachers $(M=28.87, \mathrm{SD}=4.93), t(169)=2.66, p<0.01$. Selfesteem in secondary school teachers $(M=30.53, \mathrm{SD}=5.04)$ was also significantly higher than elementary school teachers $(M=28.90, \mathrm{SD}=4.92), t(172)=2.11, p<0.05$. In addition, anxiety in secondary school teachers $(M=3.37, \mathrm{SD}=3.29)$ was significantly lower than elementary school teachers $(M=4.96, \mathrm{SD}=4.77), t(171)=-2.37, p<0.05$.

As shown in Table 1, higher self-esteem and resilience were significantly correlated to less depression, anxiety, and stress $\left(r^{2}=-0.38\right.$ to -0.53$)$. In addition, resilience and self-esteem were positively correlated with each other $\left(r^{2}=0.56\right)$. To identify predictors of the outcome variables (depression, anxiety, and depression), the assumptions (linearity and normal distribution) of multiple linear regression were met. However, multicollinearity was found. To attain a reliable prediction model, resilience and self-esteem variables were then combined as the "Strength" variable since they both might be theoretically intertwined [58]. Because of the combination, simple linear regressions were calculated to predict depression and stress. The results of the model were significant, $F(1,170)=61.62, p<0.001, R^{2}=0.27,27 \%$ of the variation in depression can be explained by the model containing Strength only $(B=-0.15$, $p<0.001)$. The predictive model was: depression $=18.08+\left(-0.15^{*}\right.$ Strength $)$. For stress, Strength was a significant predictor $(B=-0.16, p<0.001)$. The model significantly explained $20.0 \%$ of the variance in stress, $F(1,170)=41.89, p<0.001, R^{2}=0.20$. The predictive model was: stress $=21.27+(-0.16 *$ Strength $)$.

To investigate how well Strength predicts anxiety when controlling for class level, hierarchical multiple regression was used to control the confounding factor. When the class level was entered alone, it significantly predicted anxiety, $F(1,170)=5.21, p=0.02$, adjusted $R^{2}=0.02$ (only $2 \%$ of the variance in anxiety could be predicted by class level). When Strength was added, it significantly improved the prediction, $R^{2}$ change $=0.17$, $F(1,169)=36.57, p<0.001$. The entire group of variables significantly predicted anxiety, $F(2,169)=21.43, p<0.001$, adjusted $R^{2}=0.19$. However, Strength was the only significantly contributing factor to the model (Table 2 ).

\section{Discussion}

The study aimed to distinguish significant relationships among variables impacting mental health among Okinawan school teachers. Survey data revealed significant gender differences

\begin{tabular}{|c|c|c|c|c|c|c|c|c|}
\hline & $M$ & $\mathrm{SD}$ & $A$ & 1 & 2 & 3 & 4 & 5 \\
\hline 1. Depression & 4.07 & 5.30 & 0.91 & - & $0.73^{* * *}$ & $0.77^{* * *}$ & $-0.45^{* * *}$ & $-0.53^{\text {*** }}$ \\
\hline 2. Anxiety & 4.36 & 4.33 & 0.83 & & - & $0.79^{* * *}$ & $-0.38^{* * *}$ & $-0.45^{\text {*** }}$ \\
\hline 3. Stress & 6.82 & 6.34 & 0.91 & & & - & $-0.41^{* * *}$ & $-0.40^{\text {*** }}$ \\
\hline 4. Resilience & 62.71 & 14.86 & 0.94 & & & & - & $0.56^{* * *}$ \\
\hline 5. Self-esteem & 29.52 & 5.01 & 0.86 & & & & & - \\
\hline
\end{tabular}

Note(s): ${ }^{*} p<0.05, * * p<0.01, * * * p<0.001$

Table 1.

Descriptive statistics and correlations of measured variables $(N=174)$ 
JHR

35,6

Table 2.

Hierarchical multiple regression analysis summary for strength (combined resilience and self-esteem), controlling class level variable, predicting anxiety and discrepancies among mental health issues experienced by teachers responsible for varying grade levels. Resilience and self-esteem, considered together, were identified as significant predictors of depression, anxiety, and stress among this population.

Paralleling the results of previous studies on school teachers in other prefectures of Japan, the present study found that male teachers in Okinawa reported higher levels of self-esteem and better psychological wellbeing relative to their female counterparts [45,52,53]. Further, results indicated higher self-esteem and lower anxiety among high school teachers in comparison to elementary school teachers. This result supports previous studies demonstrating significant differences in the mental states of teachers appertaining to the grade levels taught [30-32]. The requirement for students to pass an entrance exam to enter high school may increase the pressures for elementary and middle school teachers to develop positive academic outcomes, thus exacerbating the mental health of such teachers [43].

In terms of resilience, the present study also supports findings from earlier research. Resilience has consistently been seen to be a predictor of mental health issues across diverse populations internationally [24]. The results of the present study similarly indicate resilience, alongside self-esteem, as significant predictors of stress within the current sample population. Self-esteem has previously demonstrated a positive correlation with resilience; thus, the corresponding relationship between resilience and self-esteem may yield a stronger impact on stress [15]. This corresponding relationship may especially be important among Japanese school teachers due to their additional responsibilities outside of student-teacher interactions. Without formal training, experienced teachers are required to mentor new teachers during their induction period, therefore, may develop Japanese teachers' resilience in overcoming the challenges that accompany their additional roles. Further, these roles may help develop self-esteem among both new and experienced teachers by cultivating new skills as a school teacher.

Similarly, the present study found that resilience and self-esteem significantly predicted depression and anxiety as illustrated in other studies [25,26]. In past research, resilience has demonstrated a positive correlation with reflective, proactive, preventative, and problemoriented coping strategies, identifying such coping strategies as beneficial components to resilience [33,34]. Thus, school teachers who possess resilience may better adapt to challenging occupational stressors, which help to reduce mental health issues such as depression and anxiety $[18,59,60]$.

\section{Implications}

As predictors of stress, anxiety, and depression, resilience and self-esteem exhibit a direct impact on an individual's psychological wellbeing. Thus, results indicate the importance of identifying methods to increase resilience and self-esteem to enhance psychological wellness. Teacher resilience is often developed through the social support provided by colleagues and

\begin{tabular}{lrrccr}
\hline Variables & $B$ & SEB & $\beta$ & $R^{2}$ & $\Delta R^{2}$ \\
\hline Step 1 & & & & 0.03 & 0.03 \\
Class level & 1.54 & 0.68 & $0.17^{*}$ & & \\
Constant & 1.88 & 1.15 & & 0.20 & 0.17 \\
Step 2 & & & & & \\
Class level & 1.05 & 0.62 & 0.12 & & \\
Strength (resilience and self-esteem) & -0.10 & 0.02 & $-0.42^{* *}$ & & \\
Constant & 11.96 & 1.15 & & & \\
Note(s): ${ }^{*} p<0.05, * * p<0.01$ & & & &
\end{tabular}


principals within schools; support from colleagues is the strongest contributor to teacher resilience [61]. As institutional leaders, principals are responsible for organizing a positive and effective school climate that supports teachers. Strong and supportive leadership by principals foster a positive school climate. Cultivating a productive school environment allows teachers the opportunity to develop healthy relationships with their students, contributing to a better understanding of their students' needs [61,62]. In turn, positive student-teacher relationships reinforce self-efficacy among teachers and increase motivation to overcome obstacles within the profession [61].

Additionally, teacher resilience increases when leaders create a supportive school environment, take an interest in teacher professional development, and administer resources needed for teachers to meet the demands of their occupation with integrity [62]. Proposing objectives to construct interpersonal relationships among all professionals within schools enhances teacher resilience and safeguards the mental state of teachers.

Several implications may be incorporated within Okinawan schools to help alleviate the stressors experienced by teachers within this particular region. The leadership role in extracurricular activities and mentorship roles occupied by experienced teachers are often mandatory within the Japanese educational system with no compensation [42]. Okinawan school teachers may benefit from creating such roles as optional, and providing compensation for taking on additional responsibilities. The compensation associated with the additional roles may increase motivation and likelihood of Okinawan teachers filling multiple roles.

In addition, the mentorship training program may bolster in benefits by being led by the principals of the institution; through this experience, principals and teachers can develop a trusting and supportive relationship, encouraging professional and personal development within Okinawan teachers and increasing teacher resilience [61].

Consistent with the research on social support, teachers in the United States reported benefits from networking with other teachers within a writing project [63]. For instance, The National Writing Project (NWP) was primarily introduced to further develop teaching techniques; simultaneously, NWP influenced self-esteem and motivation among teachers through increasing professional skills and engaging veteran teachers in their career [63]. More importantly, participation in the NWP provided a sense of belonging among the teaching community [63]. As teachers in Japan have demonstrated higher burnout among those who failed to seek help from colleagues, developing a similar project among Okinawan teachers may contribute to an increase in teacher resilience. In particular, such a project could be beneficial for teachers who experience higher anxiety.

Additionally, training teachers to implement a mental health promotion program for their students has demonstrated to indirectly increase resilience and improve psychological wellbeing among teachers [64]. Aussie Optimism, a universal mental health promotion program, was administered by teachers in Australia; the objective of this program was to focus on the risk factors of depression and anxiety among students [64]. The teachers were trained to teach social life and optimistic thinking skills to their students; these skills included problem-solving and social skills while building social support and enforcing cognitive restructuring to promote positive self-perception and self-esteem [64]. The program was effective in reducing depression and anxiety among students with elevated levels of depression; female students demonstrated decreased levels of depression and increased selfesteem [64]. More importantly, the program increased teacher resilience [64]. Initiating a mental health promotion program by focusing objectives on developing the necessary skills to decrease vulnerability to depression and anxiety may be more effective in countries with a high stigma against publicly reporting mental health concerns, such as Japan.

In the collectivistic culture of Japan, individuals experience pressure to pursue success in ways that gain the approval of their affiliates. Thus, Japanese individuals may be more self-

Resiliency and mental health of teachers 
JHR 35,6

critical. Incorporating mindfulness-based stress reduction (MBSR) may decrease negative selfperception and susceptibility to mental health issues, as demonstrated among teachers in the United States [65]. More specifically, MBSR assisted in decreasing distress associated with selfconfidence and increasing mindfulness within teachers. Additionally, MBSR interventions are available online, accommodating to the stigma against mental health issues in Japan.

Some limitations of this study included the use of cross-sectional design and convenience sampling. Although the demographic and educational characteristics of the teachers in the survey were similar to the teacher population in Okinawa, results need to be interpreted with caution. Future research should expand on this study to several prefectures in Japan to better understand similarities and differences of teacher wellbeing across different parts of Japan.

\section{Conclusions}

The present study aims to minimize the gap in the literature on the mental health of Okinawan teachers. Concurrently, it seeks to identify resilience and self-esteem in local, national, and international contexts as a means of promoting psychological wellbeing among teachers. Given the Japanese cultural emphasis on social integration, successful promotion of teacher resilience and self-esteem hinges upon the support of colleagues and principals, improved student-teacher relationships, and increased mindfulness among teachers.

\section{References}

1. Bernotaite L, Malinauskiene V. Workplace Bullying and mental health among teachers in relation to psychosocial job characteristics and burnout. Int J Occup Environ Med. 2017; 30(4): 629-40. doi: 10.13075/ijomeh.1896.00943.

2. Camacho DA, Vera E, Scardamalia K, Phalen PL. What are urban teachers thinking and feeling?. Psychol Sch. 2018; 55(9): 1133-50. doi: 10.1002/pits.22176.

3. Day C, Hong J. Influences on the capacities for emotional resilience of teachers in schools serving disadvantaged urban communities: challenges of living on the edge. Teach Teach Educ. 2016 Oct; 59: 115-25. doi: 10.1016/j.tate.2016.05.015.

4. Gray C, Wilcox G, Nordstokke D. Teacher mental health, school climate, inclusive education and student learning: a review. Can Psychol. 2017; 58(3): 203-10. doi: 10.1037/cap0000117.

5. Shernoff ES, Mehta TG, Atkins MS, Torf R, Spencer J. A Qualitative study of the sources and impact of stress among urban teachers. School Ment Health. 2011; 3(2): 59-69. doi: 10.1007/s12310011-9051-z.

6. Borrelli I, Benevene P, Fiorilli C, D'Amelio F, Pozzi G. Working conditions and mental health in teachers: a preliminary study. Occup Med (Lond). 2014 Oct; 64(7): 530-2. doi: 10.1093/occmed/ kqu108.

7. Correia FMS, de Albuquerque RN, Martins HAL, Lins LDF, Lima MDC, Dias JMS, da Silva CM, dos Santos AJ, dos Santos LT, Ribas VR. Participation in nighttime activities in the genesis of depression in public school teachers from the State of Pernambuco, Brazil. Dement Neuropsychol. 2012 Oct-Dec; 6(4): 276-85. doi: 10.1590/S1980-57642012DN06040013.

8. Hadi AA, Naing NN, Daud A, Nordin R. Work related depression among secondary school teachers in Kota Bharu, Kelantan, Malaysia. Int Med J. 2008 May; 15(2): 145-52.

9. Kosir K, Tement S, Licardo M, Habe K. Two sides of the same coin? The role of rumination and reflection in elementary school teachers' classroom stress and burnout. Teach Teach Educ. 2015; 47: 131-41. doi: 10.1016/j.tate.2015.01.006.

10. McLean L, Abry T, Taylor M, Jimenez M, Granger K. Teachers' mental health and perceptions of school climate across the transition from training to teaching. Teach Teach Educ. 2017; 65: 230-40. doi: 10.1016/j.tate.2017.03.018. 
11. McLean L, McDonald Connor C. Depressive symptoms in third-grade teachers: relations to classroom quality and student achievement. Child Dev. 2015 May-Jun; 86(3): 945-54. doi: 10.1111/ cdev.12344.

12. Ouellette RR, Frazier SL, Shernoff ES, Cappella E, Mehta TG, Marinez-Lora A, Cua G, Atkins MS. Teacher job stress and satisfaction in urban schools: disentangling individual-, classroom-, and organizational-level influences. Behav Ther. 2018 Jul; 49(4): 494-508. doi: 10.1016/j.beth.2017. 11.011.

13. Schwarzer R, Hallum S. Perceived teacher self-efficacy as a predictor of job stress and burnout: mediation analyses. Appl Psychol. 2008 Jul; 57: 152-71. doi: 10.1111/j.1464-0597.2008.00359.x.

14. Tang CSK, Au WT, Schwarzer R, Schmitz G. Mental health outcomes of job stress among Chinese teachers: role of stress resource factors and burnout. J Organ Behav. 2001 Dec; 22(8): 887-901. doi: 10.1002/job.120.

15. Kapikiran S, Acun-Kapikiran N. Optimism and psychological resilience in relation to depressive symptoms in university students: examining the mediating role of self-esteem. Educ. Sci. Theor. Pract. 2016 Dec; 16(6): 2087-110. doi: 10.12738/estp.2016.6.0107.

16. Runkewitz K, Kirchmann H, Strauss B. Anxiety and depression in primary care patients: predictors of symptom severity and developmental correlates. J Psychosom Res. 2006 May; 60(5): 445-53. doi: 10.1016/j.jpsychores.2005.09.003.

17. Sinclair VG, Wallston KA, Strachan E. Resilient coping moderates the effect of trauma exposure on depression. Res Nurs Health. 2016 Aug; 39(4): 244-52. doi: 10.1002/nur.21723.

18. Wu HC. The protective effects of resilience and hope on quality of life of the families coping with the criminal traumatisation of one of its members. J Clin Nurs. 2011 Jul; 20(13-14): 1906-15. doi: 10.1111/j.1365-2702.2010.03664.x.

19. Nezhad MAS, Besharat MA. Relations of resilience and hardiness with sport achievement and mental health in a sample of athletes. Procedia Soc Behav Sci. 2010; 5: 757-63. doi: 10.1016/j.sbspro. 2010.07.180.

20. Zimmerman MA, Arunkumar R. Resiliency research: implications for schools and policy. Soc Policy Rep. 1994; 8(4): 1-20.

21. Lu FJH, Lee WP, Chang YK, Chou CC, Hsu YW, Lin JH, Gill DL. Interaction of athletes' resilience and coaches" social support on the stress-burnout relationship: a conjunctive moderation perspective. Psychol Sport Exerc. 2016 Jan; 22: 202-9. doi: 10.1016/j.psychsport.2015.08.005.

22. Gloria CT, Faulk KE, Steinhardt MA. Positive affectivity predicts successful and unsuccessful adaptation to stress. Motiv Emot. 2013 Mar; 37(1): 185-93. doi: 10.1007/s11031-012-9291-8.

23. Richards KAR, Levesque-Bristol C, Templin TJ, Graber KC. The impact of resilience on role stressors and burnout in elementary and secondary teachers. Soc Psychol Educ. 2016 Sep; 19(3): 511-36. doi: 10.1007/s11218-016-9346-x.

24. Hao SW, Hong W, Xu HH, Zhou LL, Xie ZY. Relationship between resilience, stress and burnout among civil servants in Beijing, China: mediating and moderating effect analysis. Pers Indiv Differ. 2015 Sep; 83: 65-71. doi: 10.1016/j.paid.2015.03.048.

25. Nothling J, Ganasen K, Seedat S. Predictors of depression among a sample of South African mortuary workers. J Nerv Ment Dis. 2015 Mar; 203(3): 226-30.

26. Sharpley CF, Palanisamy SKA, Metcalf K, Jones KA, Kelly B, McFarlane JR. A comparison of a single genetic factor, two stress factors, and one psychosocial coping factor as predictors of depression in an Australian community sample. Arch. Psychiatr. Psychother. 2014; 16(4): 15-26. doi: 10.12740/APP/30099.

27. Ramanigopal CS. Self esteem and decision making styles of school teachers. J. Indian Acad. Appl. Psychol. 2008; 34(Spec Issue): 145-50.

28. Zamri EN, Moy FM, Hoe VC. Association of psychological distress and work psychosocial factors with self-reported musculoskeletal pain among secondary school teachers in Malaysia. PLoS ONE. 2017; 12(2): e0172195. doi: 10.1371/journal.pone.0172195.

Resiliency and mental health of teachers 
JHR 35,6
29. American Federation of Teachers. Educator quality of life survey. 2017 [cited 2019 Nov 11]. Available from: https://www.aft.org/2017-educator-quality-life-survey.

30. Soria-Saucedo R, Lopez-Ridaura R, Lajous M, Wirtz VJ. The prevalence and correlates of severe depression in a cohort of Mexican teachers. J Affect Disorders. 2018 Jul; 234: 109-16. doi: 10.1016/j. jad.2018.02.036.

31. Besse R, Howard K, Gonzalez S, Howard J. Major depressive disorder and public school teachers: evaluating occupational and health predictors and outcomes. J Appl Biobehav Res. 2015 Jun; 20(2): 71-83. doi: 10.1111/jabr.12043.

32. Ruijuan Y, Xuqun Y. Advancing the effort-reward imbalance model: economic rewards influence on teachers" mental health. Acta Psychologica Sinica. 2017; 49(9): 1184-94. doi: 10.3724/sp.J.1041. 2017.01184.

33. Ishikawa H, Kawakami N, Kessler RC, World Mental Health Japan Survey Collaborators. Lifetime and 12-month prevalence, severity and unmet need for treatment of common mental disorders in Japan: results from the final dataset of World Mental Health Japan Survey. Epidemiol Psychiatr Sci. 2016 Jun; 25(3): 217-29. doi: 10.1017/S2045796015000566.

34. World Health Organization [WHO]. Depression and other common mental disorders: global health estimates. [cited 2019 Nov 1]. Available from: https:/apps.who.int/iris/bitstream/handle/10665/ 254610/WHO-MSD-MER-2017.2-eng.pdf.

35. Ibrahim AK, Kelly SJ, Glazebrook C. Analysis of an Egyptian study on sthe socioeconomic distribution of depressive symptoms among undergraduates. Soc Psych Psych Epid. 2012 Jun; 47(6): 927-37. doi: 10.1007/s00127-011-0400-x.

36. Roberts R, Golding J, Towell T, Weinreb I. The effects of economic circumstances on British students' mental and physical health. J Am Coll Health. 1999 Nov; 48(3): 103-9. doi: 10.1080/ 07448489909595681.

37. Statistics Japan. Correlation with Okinawa. [cited 2019 Nov 1]. Available from: https://stats-japan. com/t/tdfk/okinawa.

38. Okinawa Prefectural Government. Introduction of Okinawa. [updated 2013 Dec 23; cited 2020 Jan 19]. Available from: https://www.pref.okinawa.jp/site/chijiko/kohokoryu/foreign/english/ introduction/index.html.

39. Manalo E, Kusumi T, Koyasu M, Michita Y, Tanaka Y. To what extent do culture-related factors influence university students' critical thinking use?. Think Skills Creat. 2013; 10: 121-32. doi: 10. 1016/j.tsc.2013.08.003.

40. Bullard EW, Hosoda T. Help-seeking behavior for depression in Japanese schoolteachers. Int J Ment Health. 2015; 44(3): 169-85. doi: 10.1080/00207411.2015.1035058.

41. Hatta T, Nishiide S. Teachers' stress in Japanese primary schools: comparison with workers in private companies. Stress Med. 1991; 7(4): 207-11. doi: 10.1002/smi.2460070403.

42. National Center on Education and the Economy. Japan: teacher and principal quality. [cited 2020 Jan 19]. Available from: http://ncee.org/what-we-do/center-on-international-educationbenchmarking/top-performing-countries/japan-overview/japan-teacher-and-principal-quality/.

43. Kids Web Japan. Explore Japan: schools. [cited 2020 Jan 19]. Available from: https://web-japan.org/ kidsweb/explore/schools/.

44. Takahara R. Public school teachers'use of sick leave due to mental illness: multilevel structural equation modeling using individual and prefecture-level data. Jpn. J. Educ. Psychol. 2015; 63(3): 242-53. doi: 10.5926/jjep.63.242.

45. Nagai M, Tsuchiya KJ, Toulopoulou T, Takei N. Poor mental health associated with job dissatisfaction among school teachers in Japan. J Occup Health. 2007 Nov; 49(6): 515-22. doi: 10.1539/joh.49.515.

46. Chen H. Stress and life events of middle school teachers. Chin. Ment. Health J. 2002; 16(8): 527-28.

47. Leroux M, Theoret M. Intriguing empirical relations between teachers' resilience and reflection on practice. Reflective Pract. 2014; 15(3): 289-303. doi: 10.1080/14623943.2014.900009. 
48. Munakata T, Shiiya J. Burnout of junior high school teachers and their psycho-social environment. J. Ment. Health. 1986; 33: 129-53.

49. Doi K, Hashiguchi $\mathrm{H}$. The relationship between irrational belief and mental health in junior highschool teachers. Jpn. J. Health Psychol. 2000; 13(1): 23-30. doi: 10.11560/jahp.13.1_23.

50. Tanaka N, Uji M, Hiramura H, Chen Z, Shikai N, Kitamura T. Cognitive patterns and depression: study of a Japanese university student population. Psychiatry Clin Neurosci. 2006 Jun; 60(3): 358-64. doi: 10.1111/j.1440-1819.2006.01514.x.

51. Nishizaka S. Kindergarten teachers' mental health: stress, pre-school teacher efficacy, and hardiness. Jpn. J. Educ. Psychol. 2002 Sep; 50(3): 283-90. doi: 10.5926/jjep1953.50.3_283.

52. Nishida J, Otomo S. Influence of exercise and physical activity on the mental health of elementary and junior high school teachers: individual characteristics and experiences with stress. Jpn. J. Educ. Psychol. 2010 Sep; 58(3): 285-97. doi: 10.5926/jjep.58.285.

53. Tamura S, Ishikuma T. Help-seeking preferences and burnout: junior high school teachers in Japan. Jpn. J. Educ. Psychol. 2001 Dec; 49(4): 438-48. doi: 10.5926/jjep1953.49.4_438.

54. Lovibond SH, Lovibond PF. Manual of the depression anxiety stress scales. $2^{\text {nd }}$ ed. Sydney: Psychology Foundation; 1995.

55. Ito M, Nakajima S, Shirai A, Kim Y. Cross-cultural validity of the Connor-Davidson Scale: data from Japanese population. Poster presented at: 25th Annual Meeting, International Society of Traumatic Stress Studies (ISTSS); 2009 Nov; Atlanta, GA.

56. Mimura C, Griffiths P. A Japanese version of the Rosenberg Self-Esteem Scale: translation and equivalence assessment. J Psychosom Res. 2007 May; 62(5): 589-94. doi: 10.1016/j.jpsychores.2006.11.004.

57. Faul F, Erdfelder E, Buchner A, Lang AG. Statistical power analyses using G*Power 3.1: tests for correlation and regression analyses. Behav Res Methods. 2009 Nov; 41(4): 1149-60. doi: 10.3758/BRM.41.4.1149.

58. Leech NL, Barrett KC, Morgan GA. SPSS for intermediate statistics: use and interpretation. $3^{\text {rd }}$ ed. New York: Taylor \& Francis; 2008.

59. Li WW, Miller DJ. The impact of coping and resilience on anxiety among older Australians. Aust J Psychol. 2017 Dec; 69(4): 263-72. doi: 10.1111/ajpy.12152.

60. Wu LM, Sheen JM, Shu HL, Chang SC, Hsiao CC. Predictors of anxiety and resilience in adolescents undergoing cancer treatment. J Adv Nurs. 2013 Jan; 69(1): 158-66. doi: 10.1111/j.1365-2648.2012.06003.x.

61. Greenfield B. How can teacher resilience be protected and promoted?. Educ. Child Psychol. 2015; 32(4): 52-68.

62. Peters J, Pearce J. Relationships and early career teacher resilience: a role for school principals. Teach Teach. 2012; 18(2): 249-62. doi: 10.1080/13540602.2012.632266.

63. Yonezawa S, Jones M, Singer NR. Teacher resilience in urban schools: the importance of technical knowledge, professional community, and leadership opportunities. Urban Educ. 2011 Sep; 46(5): 913-31. doi: 10.1177/0042085911400341.

64. Tyson O, Roberts CM, Kane R. Can implementation of a resilience program for primary school children enhance the mental health of teachers? Aust. J. Guid. Counsell. 2009; 19(2): 116-30. doi: 10. 1375/ajgc.19.2.116.

65. Gold E, Smith A, Hopper I, Herne D, Tansey G, Hulland C. Mindfulness-based stress reduction (MBSR) for primary school teachers. J Child Fam Stud. 2010 Apr; 19(2): 184-9. doi: 10.1007/s10826-009-9344-0.

\section{Corresponding author}

Paul Ratanasiripong can be contacted at: paul.ratanasiripong@csulb.edu

For instructions on how to order reprints of this article, please visit our website:

www.emeraldgrouppublishing.com/licensing/reprints.htm

Or contact us for further details: permissions@emeraldinsight.com
Resiliency and mental health of teachers 